\title{
Erratum to: A generalized Jentzsch theorem
}

\author{
A. K. Kitover
}

Published online: 10 May 2011

(C) Springer Basel AG 2011

\section{Erratum to: Positivity (2005) 9:501-509 DOI 10.1007/s11117-004-8291-7}

There is an error in the proof of Lemma 10 in the above-mentioned paper. The implication that operator $T$ leaves invariant the principal band generated by the element $z$ because it leaves invariant the principal ideal generated by $z$ cannot be justified because we do not assume $T$ to be $\sigma$-order continuous. I am grateful to Anton Schep who indicated to me the said error. The following changes have to be made.

(1) In part ( $a$ ) of Theorem 6 the operator $R$ should be assumed order continuous instead of just $\sigma$-order continuous. (I do not know if the result remains true under the milder condition of $\sigma$-order continuity of $R$ )

(2) The proof of Lemma 10 then goes as follows:

Let us prove first that $T$ is $\sigma$-order continuous. Let $x_{n} \downarrow 0$ then $R x_{n} \downarrow 0$ and $R x_{n} \stackrel{w}{\rightarrow} 0$ whence $S x_{n} \downarrow 0$ and $S x_{n} \stackrel{w}{\rightarrow} 0$. Assume contrary to what we claim that $T x_{n} \geq y \geq 0$ and $y \neq 0$. Then $S T x_{n} \geq S y$ and $S T x_{n} \leq T S x_{n} \stackrel{w}{\rightarrow} 0$. Therefore $S y=0$. Let $Z$ be the maximal by inclusion ideal in $X$ such that $S \mid Z=0$. $Z$ obviously exists by Zorn's lemma. Then $Z$ is a band because $S$ is order-continuous. We claim that $T Z \subseteq Z$. Indeed, otherwise there is a positive $u \in Z$ such that $T u \notin Z$. But $S T u \leq T S u=0$ and we come to a contradiction with the maximality of $Z$. The remaining part of the proof does not have to be changed.

The online version of the original article can be found under doi:10.1007/s11117-004-8291-7.

A. K. Kitover $(\varangle)$

Department of Mathematics, Community College of Philadelphia,

1700 Spring Garden Street, Philadelphia, PA 19130, USA

e-mail: akitover@ccp.edu

Birkhäuser 